Relations industrielles

Industrial Relations

\title{
La situation syndicale dans la Fédération de Malaisie. Rapport d'une mission du Bureau international du travail. Bureau international du travail, Genève, 1962. 120 pages.
}

\section{Gérard Dion}

Volume 17, numéro 4, octobre 1962

URI : https://id.erudit.org/iderudit/1021492ar

DOI : https://doi.org/10.7202/1021492ar

Aller au sommaire du numéro

\section{Éditeur(s)}

Département des relations industrielles de l’Université Laval

\section{ISSN}

0034-379X (imprimé)

1703-8138 (numérique)

Découvrir la revue

Citer ce compte rendu

Dion, G. (1962). Compte rendu de [La situation syndicale dans la Fédération de Malaisie. Rapport d'une mission du Bureau international du travail. Bureau international du travail, Genève, 1962. 120 pages.] Relations industrielles / Industrial Relations, 17(4), 505-507. https://doi.org/10.7202/1021492ar

Tous droits réservés @ Département des relations industrielles de l’Université Laval, 1963
Ce document est protégé par la loi sur le droit d'auteur. L’utilisation des services d'Érudit (y compris la reproduction) est assujettie à sa politique d'utilisation que vous pouvez consulter en ligne.

https://apropos.erudit.org/fr/usagers/politique-dutilisation/ 
sition diamétralement opposée et que les Etats-Unis ont cherché à concilier les humanités, les sciences et l'organisation. Il constate aussi une énorme confusion dans la présentation des statistiques parce que les divers pays n'ont pas un dénominateur commun pour définir ce qu'est l'enseignement supérieur, etc. Il critique vertement la pratique de certains pays qui pour des motifs de prestige national ou de propagande politique faussent les statistiques éducatives.

L'auteur dénote une grande érudition ainsi qu'une connaissance théorique et factuelle très poussée du problème de l'éducation. Son analyse s'appuie sur une documentation considérable et très diversifiée qu'il décortique avec perspicacité et sagacité en soulignant les erreurs auxquelles il apporte les corrections appropriées.

Sans être spécialiste des questions de l'éducation, nous sommes persuadés que les personnes dont c'est la sphère d'activité apprécieront beaucoup «Sociologie de l'éducation » non seulement à cause de l'ampleur de sa documentation, mais aussi, à cause des problèmes qu'il découvre et du défi qu'il lance à nos civilisations modernes.

\section{L.-M. Tremblay}

\section{Statistiques de la Province de Québec,} Ministère de l'Industrie et du Commerce, Division des études spéciales, Bureau de la Statistique du Québec, vol. I, no 6, sept. 1962.

Il y a quelques mois, le Bureau de la Statistique du Québec, lançait une nouvelle revue consacrée à la présentation unifiée des principales statistiques sur la province de Québec. Le contenu du sixième numéro, paru en septembre 1962, indique que cette revue a adopté sa forme définitive après avoir, à l'occasion du premier numéro, fait appel aux suggestions et aux critiques des lecteurs. Le temps est donc venu d'en faire une brève appréciation.

On ne saurait, en premier lieu, trop féliciter et encourager les responsables de cette heureuse initiative; l'honorable André Rousseau et monsieur René Tremblay respectivement ministre et sous-ministre au ministère du Commer$\mathrm{ce}$ et de l'Industric. Bien que ne contenant pas d'informations inédites, cette revue constitue un excellent instrument de travail qui répond à un besoin, parce qu'elle réunit dans une même publication les données essentielles sur la province.

Le dernier numéro contient un intéressant éditorial sur la situation conjoncturelle de la province de Québec. Nous souhaitons que cette pratique diun article de fond se continue et même que l'on y accorde encore plus d'espace.

L'information statistique se divise en quatre parties. La première couvre les principaux indicateurs économiques. La seconde touche le monde du travail : emploi, salaires, revenus, chômage ainsi que les grèves et lock-out. Personnellement, nous accepterions plus d'informations syndicales, telles que le nombre et la répartition des effectifs syndicaux. La troisième traite des prix, de la production industrielle et manufacturière ainsi que de la construction. La dernière, plus diversifiée, porte sur l'agriculture et Palimentation, le commerce, le transport et la finance.

Comme nous l'avons dit précédem. ment, cette revue ne tire pas son originalité des informations qu'elle contient. La plupart des statistiques sont empruntées au B.F.S. : comptes nationaux, revue statistique du Canada, emploi et salaires, rapport statistique sur l'application de la loi sur l'assurance-chômage, prix et indices des prix, etc. Le reste se retrouve dans les diverses publications du Bureau de la Statistique du Québec. L'originalité provient plutôt de la réunification des diverses données, de leur présentation comparative avec l'Ontario et le Canada ainsi que des divers graphiques qui permettent en un coup d'oeil d'avoir une idée claire et rapide de la situation dans chaque cas.

Nous sommes persuadés que ceux qui sont appelés à recourir à ce genre d'informations statistiques, $y$ trouveront un bon instrument de travail, qui favorise la confrontation rapide de données diverses.

\section{L.-M. Tremblay}

La situation syndicale dans la Fédération de Malaisie. Rapport d'une mission du Bureau international du travail. Bureau international du travail, Genève, 1962. 120 pages.

Dans le cadre des enquêtes sur la pratique de la liberté syndicale entre- 
Relations Industritelles, vol. 17, No 4

prises par le Bureau international du travail, une mission officielle de cinq membres a séjourné du 28 septembre au 28 octobre 1961 dans la Fédération Malaise. Cet ouvrage constitue le rapport de la Mission.

Comme dans toutes les enquêtes conduites jusqu'ici (aux Etats-Unis, en U.R.S.S., en Grande-Bretagne, en Suède), la mission a été amenée à étudier le contexte historique du développement social, économique et politique, le cadre juridique et le régime des rapports collectifs du travail, Porganisation syndicale, l'organisation patronale, les réactions du gouvernement, des employeurs, des travailleurs et du public vis-à-vis du syndicalisme ouvrier.

Evidemment chaque pays possède des caractéristiques particulières. Mais ce qu'il y a de significatif et aussi d'intéressant dans cette enquête, c'est qu'elle portait sur un petit pays de six millions d'habitants qui vient d'accéder à l'indépendance. En effet, la Fédération de Malaisie ne possède son autonomie politique que depuis le 31 août 1957. Elle est un pays qui n'a pas encore achevé son processus de formation. La moitié des habitants seulement est d'origine malaisienne, trente-sept pour cent sont d'origine chinoise et dix pour cent sont d'origine indienne. Ce mélange racial et culturel avec la multiplicité des langues a une influence sur l'organisation syndicale tout comme le fait que le quart seulement de la population réside dans les villes. De plus, la moitié de la maind'oeuvre est encore occupée dans l'agriculture (grandes plantations), deux pour cent dans les mines et carrières et huit pour cent dans l'industrie, commerce, services administratifs, etc.

L'histoire récente semble aussi avoir encore une grande influence sur le régime syndical et les attitudes du gouvernement et des employeurs.

La Malaisie, depuis le siècle dernier jusqu'à son indépendance faisait partie de l'empire britannique. On n'a pas à se surprendre si le régime juridique et la forme d'organisation syndicale s'inspirent grandement des institutions de la Grande-Bretagne. La juridiction du travail relève du gouvernement central qui possède un ministère du travail.

Ce pays a connu l'occupation japonaise au cours de la dernière guerre mondiale. A la fin des hostilités, profi- tant d'un désir général de liberté et d'une prise de conscience nationale, les communistes s'efforcèrent d'accroître leur influence dans le pays et de conquérir le contrôle de l'économie en travaillant dans le mouvement syndical. La multiplication des grèves, la violence et même des guerres d'embuscade contre les dirigeants des plantations, des mines et autres industries amenèrent le gouvernement à prendre des mesures énergiques qui freinèrent le mouvement syndical.

Aussi, la création des syndicats et l'organisation syndicale elle-même sontelles soumises encore à une règlementation assez sévère et à des contrôles qui visent à empêcher toute influence extérieure dans le mouvement ouvrier. Le Registrar, chez qui doivent s'enregistrer tous les spndicats sous peine d'être dans l'illégalité, jouit de pouvoirs de surveillance très étendus. Les syndicats ne peuvent grouper que des travailleurs d'une même profession ou d'une profession similaire ou d'une même industrie. Il en résulte une multiplicité de petits syndicats sans grande force. Il existe cependant trois grandes fédérations : la Fédération des syndicats d'enseignants, la Fédération panmalaise des syndicats chinois de l'industrie mécanique et le Congrès des syndicats des services publics et de l'administration. Le seul organisme central, le Congrès de syndicats de Malaisie, représente la grande masse des travailleurs, mais ne détient aucun pouvoir exécutif et ne peut être enregistré comme syndicat; il joue un rôle consultatif et coordinateur.

La base du régime des relations industrielles s'inspire du principe des accords volontaires. Le développement syndical, quoiqu'encouragé par le gouvernement - il y a même au sein du ministère du travail une section chargée d'aider, de conseiller et de guider les syndicats est handicappé par suite de la nature de l'emploi, de la diversité ethnique chez les travailleurs, de leur niveau d'instruction et aussi, dans une certaine mesure, de l'attitude des employeurs. Chez ceux-ci, il s'en trouve qui acceptent le fait syndical, mais d'autres seraient prêts à favoriser les négociations collectives candition de trouver des syndicats capables d'assumer leur part de responsabilités et dirigés par des hommes intègres, compétents et soucieux de se tenir à l'écart des luttes politiques » (p. 92). Ce sont là des condi- 
tions à remplir qui rappellent des refrains déjà connus. Et lon sait ce que cela veut dire.

Cinq ans d'expérience syndicale dans un pays en pleine transformation économique et sociale, c'est bien peu. Mais il est intéressant de voir comment à partir d'une conception de la liberté, comme nous l'entendons dans le monde occidental, le syndicalisme est en train de s'organiser avec la sympathie du gouvernement. Ce rapport est une contribution utile à l'étude du syndicalisme et des relations industrielles dans le monde.

\section{Gérard Dion}

\section{Principes déconomie politique, par Ro-} ger Dehem, Dunod, Paris 1962, pp. 189.

Ce n'est pas tous les jours qu'un professeur de l'Université Laval publie un livre sérieux sur l'économie. C'est un évènement rare qu'il vaut la peine de signaler.

Le livre du professeur Dehem s'adresse à ceux qui s'intéressent aux grands problèmes économiques de notre temps. Il ne s'adresse pas qu'à ceux dont l'étude des phénomènes économiques est l'occupation continuelle. Tous ceux que touchent les problèmes de l'élimination de la misère o ou \&l'élimination du bien-être de la société \$ et qui veulent en amorcer une étude sérieuse auront profit à lire les « Principes d'économie politique ».

Le livre, bien qu'il soit un livre d'initiation, bien que l'auteur, pour des raisons pédagogiques, se soit abstenu d'utiliser les techniques d'analyse habituelles aux économistes, ne va pas sans difficulté. L'Auteur a échappé à la tentation de rendre trop facile ce qui ne l'est pas en risquant de perdre l'essentiel. C'est pourquoi son livre est à la fois élémentaire et approfondi. Dans ce dernier sens, c'est un livre difficile. Mais la connaissance des phénomènes économiques et des règles qui les régissent l'est aussi.

N'importe lequel lecteur se sent en terrain connu, lorsque l'auteur réfère, par exemple, à cet anglais Child comme à «un précurseur typique des plaideurs de politique économique en fonction d'intérêts particuliers 》; ou lorsqu'il rappelle que les phénomènes économiques sont liés \& non seulement aux éléments atmosphériques, mais aussi à l'échelonnement d'événements sociaux telles les fêtes de Noël et de Pâques 》. Le même lecteur se sent cependant moins à l'aise lorsque l'auteur parlera sans explication supplémentaire de $<$ la violation des conditions d'un optimum parétien $\gg$, (p. 160) ou de la démonstration faite par certains auteurs du fait que * si l'épargne pouvait être tellement abondante que sa productivité marginale descende à zéro, la «productivité sociale» de l'économie serait maxima ». (p. 159).

Mais ces passages plus difficiles devraient être pour le lecteur une invitation à un approfondissement des concepts auxquels sont habitués les économistes, lesquels concepts sont nécessaires à la compréhension des phénomènes économiques.

Cet approfondissement est rendu facile par les excellentes références qui apparaissent à la fin de chaque chapitre et qui ont le mérite d'être sûres et de n'être pas trop nombreuses.

Signalons enfin que le style est correct et concis et que le vocabulaire est moderne ( guidage », 《retombées 》, « la nouvelle vague \$, « déviation conservatrice $\gg$ ).

Le livre de M. Dehem n'est pas un livre parfait. Mais c'est un livre honnête et sérieux. Il a le mérite de ne pas éloigner les non-initiés par une surcharge de preuves géométriques ou de relations mathématiques; d'apporter aux facteurs institutionnels et politiques plus d'importance qu'en accordent les économistes; de ne pas esquiver la complexité d'une analyse qui conduit à des solutions possibles mais pas toujours certaines; de rendre évident que les problèmes économiques ne peuvent pas être résolus en disant <hocus pocus $\gg$ ou par quelques gestes de magiciens.

Nous souhaitons, avec l'auteur, que ce livre conduise à d'autres.

\section{Jacques St-Laurent}

The Economics of Trade Unions, by Albert Rees, University of Chicago Press, 1962, pp. 202.

Voici le dernier venu de l'excellente collection « Cambridge Economic Hand- 